\title{
Implantes en pacientes VIH-positivo. A propósito de tres casos
}

\section{Dental implants in HIV-positive patients. A presentation of three cases}

\author{
FERRÚS-TORRES E* \\ PIÑERA-PENALVA IM** \\ BERINI-AYTÉS L*** \\ GAY-ESCODA C***
}

\author{
Ferrús-Torres E, Piñera-Penalva M, Berini-Aytés L, Gay-Escoda C. \\ Implantes en pacientes VIH-positivo. A propósito de tres casos. Av \\ Periodon Implantol. 2008; 20, 3: 165-172.
}

\begin{abstract}
RESUMEN
El aumento de la calidad y la esperanza de vida de los pacientesVIH-positivo, ha hecho que la solicitud de tratamiento estético dental se haya incrementado significativamente en estos pacientes, considerándose actualmente las prótesis implantosoportadas como una opción terapéutica válida.

Se presentan 3 pacientes varones, VIH-positivo que acudieron a nuestro Servicio de Implantología Bucofacial, para valorar una posible rehabilitación implantosoportada. La evolución clínica de los pacientes había sido estable durante un periodo mínimo de 4 años. Se colocaron un total de 12 implantes (caso 1: 4 implantes Defcon ${ }^{\circledR}, 2$ en maxilar superior y 2 en mandíbula; caso $2: 2$ implantes ITI ${ }^{\circledR}$ en mandíbula; caso 3: 6 implantes Brånemark System ${ }^{\circledR}$ en maxilar superior), todos ellos bajo anestesia locorregional y siguiendo la técnica habitual. No se llevó a cabo ningún tipo de técnica de regeneración ósea guiada. No se presentaron complicaciones intra y/o postoperatorias. Tampoco se produjo ninguna alteración de los parámetros biológicos de control de la enfermedad de base tras la intervención quirúrgica. Los implantes han sido considerados como una opción de tratamiento en este tipo de pacientes pese a que su fiabilidad, no ha sido todavía establecida a largo plazo. A excepción de dos artículos (implante unitario inmediato y una rehabilitación bucal completa), ningún otro estudio ha sido publicado referente a la rehabilitación con implantes en pacientes VIH-positivo. En ambos artículos se corrobora la hipótesis que la cirugía bucal menor no aumenta el riesgo de infección locorregional en los pacientes VIH-positivo correctamente controlados, como evidenciamos en nuestros 3 casos. En el postoperatorio inmediato, no se observó ninguna disminución significativa del marcador $\mathrm{CD}_{4}$ ni de ningún otro parámetro biológico, tal como se describe en la literatura, obteniéndose una excelente curación de los tejidos blandos y duros.
\end{abstract}

PALABRAS CLAVE: Infección por HIV, implantes dentales, implantología bucofacial.

\section{SUIMIMARY}

The improvement in survival and quality of life of HIV-positive patients has led to a significant rise in demand for esthetic dental treatment among these patients. In this context, implant-supported prostheses are currently considered a valid treatment option.

We present three HIV-positive males seen in our Service of Oral Implantology for the evaluation of possible implant-supported rehabilitation. The clinical course of the patients had remained stable

* Odontólogo. Residente del Máster de Cirugía Bucal e Implantología Bucofacial. Facultad de Odontología. de la Universidad de Barcelona.

** Médico Estomatólogo. Profesor Asociado de Cirugía Bucal. Profesor del Máster de Cirugía Bucal e Implantología Bucofacial. Facultad de Odontología de la Universidad de Barcelona. Investigador del IDIBELL.

*** Doctor en Medicina. Estomatólogo. Especialista en Cirugía Maxilofacial. Profesor Titular de Patología Quirúrgica Bucal y Maxilofacial. Profesor del Máster de Cirugía Bucal e Implantología Bucofacial. Facultad de Odontología de la Universidad de Barcelona. Investigador del IDIBELL.

**** Doctor en Medicina. Estomatólogo. Especialista en Cirugía Maxilofacial. Catedrático de Patología Quirúrgica Bucal y Maxilofacial. Director del Máster de Cirugía Bucal e Implantología Bucofacial. Facultad de Odontología de la Universidad de Barcelona. Investigador Coordinador del IDIBELL. Jefe del Servicio de Cirugía Bucal, Implantología Bucofacial y Cirugía Maxilofacial del Centro Médico Teknon. Barcelona. 
for a minimum of four years. A total of 12 implants were positioned (case 1: 4 Defcon ${ }^{\circledR}$ implants, 2 in the upper maxilla and 2 in the mandible; case 2: 2 ITI ${ }^{\circledR}$ implants in the mandible; and case 3: 6 Brånemark System ${ }^{\circledR}$ implants in the upper maxilla;), all under locoregional anesthesia and applying the usual technique. No guided bone regeneration procedures were applied. There were no intraand/or postoperative complications, and no alterations in the biological parameters of background disease control following the surgical intervention.

Implants have been considered a treatment option in patients of this kind, despite the fact that their long-term reliability has not been established. With the exception of two articles (respectively involving immediate singe implant placement and complete oral rehabilitation), no other study has been published on rehabilitation with implants in HIV-positive patients. Both of these articles corroborate the hypothesis that minor oral surgery does not increase the risk of locoregional infection in correctly controlled HIV-positive patients, as reflected in our three cases. In the immediate postoperative period, there were no significant variations in CD4+ cell count or in any other biological parameter, as described in the literature, with excellent soft and hard tissue healing.

KEY WORDS: HIV infection, dental implants, orofacial implantology.

Fecha de recepción: 9 de abril de 2008.

Fecha de aceptación: 6 de mayo de 2008.

\section{INTRODUCCIÓN}

En los años ochenta, se desató una cierta alarma social ante los efectos de una enfermedad que era nueva y desconocida (1). Se diagnostica en España el primer caso de SIDA en el año 1981, más concretamente en la ciudad de Barcelona (2). Desde ese momento se produce un incremento significativo de notificaciones de esta enfermedad en todo el mundo, tanto del número de muertes, como el de nuevos pacientes infectados. No será hasta aproximadamente el año 1996, cuando con la introducción generalizada de los tratamientos antirretrovirales, se produzca por primera vez una disminución tanto del número de muertes como de nuevos casos. Según datos de la Organización Mundial de la Salud (OMS), actualmente más de 60 millones de personas están infectadas por el Virus de la Inmunodeficiencia Humana (VIH) , siendo la primera causa de muerte en África subsahariana y cuarta en todo el mundo. (2)

Afortunadamente, la evolución de estos pacientes ha cambiado mucho desde el inicio de la pandemia. El aumento tanto de la calidad como de la esperanza de vida de estos pacientes, con la implantación de los nuevos protocolos terapéuticos han convertido esta enfermedad en una patología crónica. Todo ello ha producido un incremento significativo de las demandas de tratamiento estético dental y funcional de estos pacientes, considerándose actualmente las prótesis implantosoportadas como una alternativa de tratamiento a las prótesis removibles que estaban condenados a soportar. Sin embargo, todavía no disponemos de una experiencia amplia fiable ni clínica ni experimental y existe poca literatura al respecto. El objetivo de nuestro trabajo es mostrar nuestra experiencia en el tratamiento mediante una rehabilitación bucodentaria implantosoportada en pacientes VIHpositivo y valorar los parámetros a tener en cuenta por parte del cirujano bucal, en el estudio preoperatorio.

\section{CASOS CLÍNICOS}

Se presentan 3 casos clínicos de pacientes VIH-positivo sometidos a un tratamiento implantosoportado en nuestro Servicio de Implantología Bucofacial del Máster de Cirugía Bucal e Implantología Bucofacial de la Universidad de Barcelona.

El periodo de estudio abarcó desde el mes de Junio de 2004 hasta el mes de Septiembre de 2006. Los 3 pacientes VIH-positivo acudieron a nuestro Servicio, para valorar la posibilidad de una rehabilitación implantosoportada.

La interconsulta entre el cirujano bucal y el médico especialista encargado del control de la enfermedad crónica, fue estrecha durante toda la planificación del tratamiento.

Para el estudio preoperatorio, se solicitaron a todos los pacientes las siguientes parámetros biológicos: estudio básico de la hemostasia, bioquímica sanguínea, biometría hemática, recuento de las subpoblaciones linfocitarias $\left(\mathrm{CD}_{4}, \mathrm{CD}_{8}\right.$, cociente $\left.\mathrm{CD}_{4} / \mathrm{CD}_{8}, \mathrm{NK}\right)$ y la carga viral. 
Todas las intervenciones quirúrgicas se llevaron a cabo bajo anestesia locorregional, y siguiendo la técnica clásica en dos fases. En ninguno de nuestros pacientes, se llevó a cabo ningún tipo de técnica de regeneración ósea guiada. Los tres pacientes, recibieron profilaxis antibiótica (amoxicilina l gramo por vía oral una hora antes de la intervención quirúrgica).

La medicación postoperatoria administrada fue igual para todos los pacientes: $750 \mathrm{mg}$ de amoxicilina (Clamoxyl ${ }^{\circledR}$, GlaxoSmithKline, Madrid, España) 1 comprimido cada 8 horas durante 7 días, $50 \mathrm{mg}$ de diclofenaco ( Diclofenaco Llorens ${ }^{\circledR}$, Llorens, Barcelona, España), 1 comprimido cada 8 horas durante 5 días, $575 \mathrm{mg}$ de metamizol (Nolotil ${ }^{\circledR}$, Boehringer Ingelheim, Barcelona, España), 1 comprimido cada 6 horas durante 3-4 días si presentaba dolor, y digluconato de clorhexidina al 0,12\% en colutorio (Clorhexidina Lacer ${ }^{\circledR}$, Lacer, Barcelona, España), 2 enjuagues al día durante 15 días. No se produjeron complicaciones intra o postoperatorias en ningún caso. No se observaron problemas en la curación de los tejidos blandos y/o duros.

No se produjo ninguna alteración de los parámetros biológicos de control de la enfermedad de base tras la intervención quirúrgica, ni durante el tiempo de seguimiento del paciente.

\section{Caso 1}

Paciente varón de 45 años, diagnosticado de VIH en el año 1998. La vía de contagio fue por adicción a drogas por vía parenteral (ADVP). Como antecedentes patológicos asociados, el paciente presentó infección por tuberculosis en el mismo año que fue diagnosticado de VIH, tratada durante 9 meses mediante tuberculostáticos, actualmente presentaba como secuela de la infección tuberculosa, una enfermedad granulomatosa gástrica desde hacía 4 años tratada con prednisona a una dosis de $20 \mathrm{mg} /$ día. El tratamiento antirretroviral que sigue el paciente desde el diagnóstico de su enfermedad consiste en $150 \mathrm{mg}$ de lamivudina (3TC) y $300 \mathrm{mg}$ de zidovudina (AZT) (Combivir ${ }^{\circledR}$, Glaxo SmithKline, Madrid, España) y $200 \mathrm{mg}$ de nevirapina (Viromune ${ }^{\circledR}$, Boehringer Ingelheim, San Cugat del Vallés, España). El paciente mantenía cifras de $\mathrm{CD}_{4}$ entre 25 y $30 \%$ y una carga viral indetectable. La cifra de plaquetas era normal. El paciente no presentaba alergias medicamentosas conocidas. El diagnóstico odontológico fue de edentulismo parcial que afectaba a los dientes $1,5,1,6,3,6$ y 4,6. La causa de las pérdidas dentarias había sido por caries hacía aproximadamente 2 años. El plan de tratamiento que se decidió, consistía en colocar 4 coronas metal-cerámica unitarias sobre implantes Defcon ${ }^{\circledR}$ (Impladent, Sabadell, España), 2 en el maxilar superior y 2 en la mandíbula (4 implantes TSA 3,0x13 mm) (Figura 1). La carga de los implantes se realizó a los 6 meses de su inserción y el tiempo de seguimiento ha sido de 18 meses.

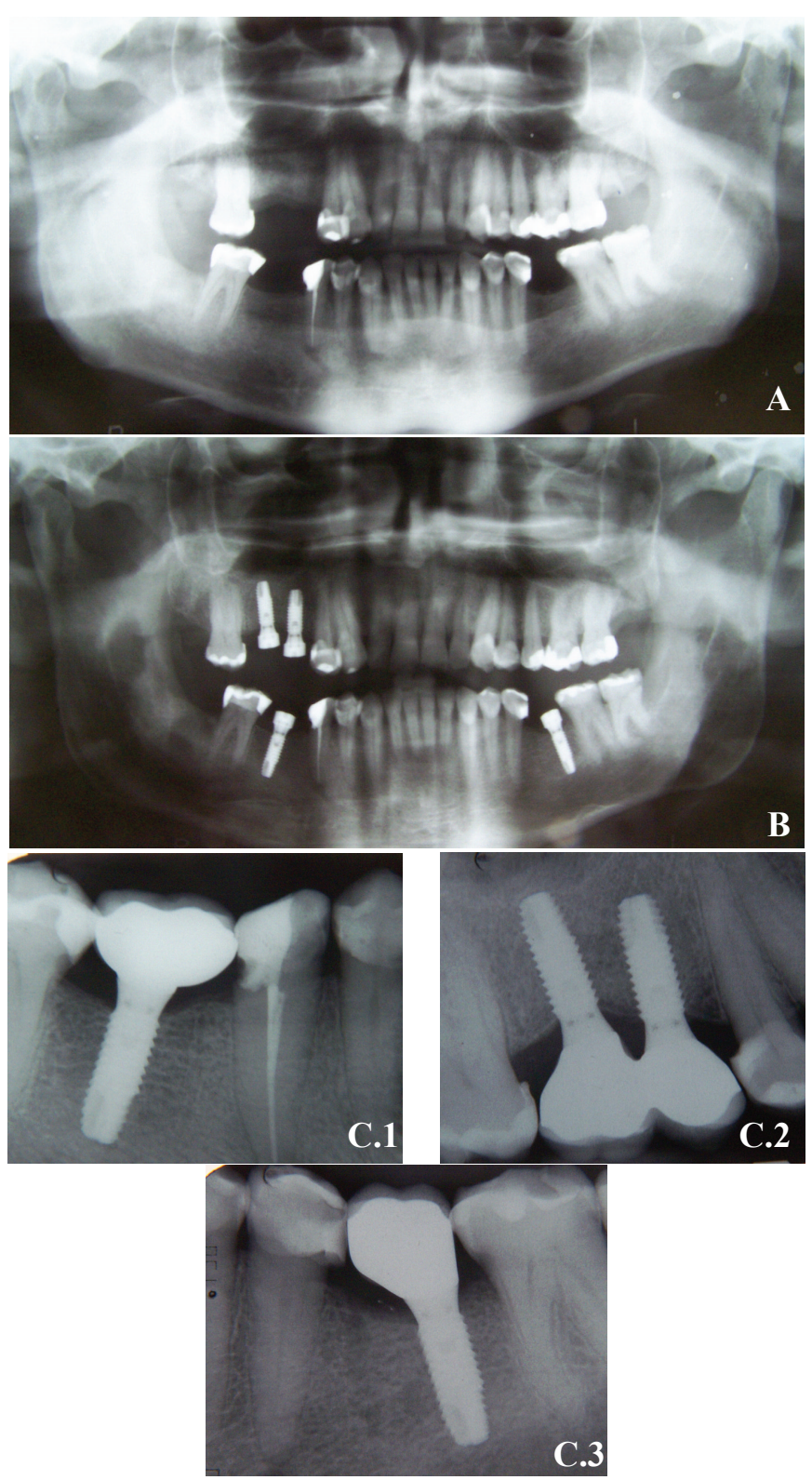

Fig. 1: Caso clínico 1. (A) Ortopantomografía que muestra los espacios edéntulos del 1,5, 1,6, 3,6 y 4,6. (B) Ortopantomografía realizada a los 6 meses tras la inserción de los 4 implantes Defcon ${ }^{\circledR}(4 \mathrm{x}$ TSA 3,0x13 mm). (C) Rehabilitación implantosoportada finalizada. C.1) Radiografía periapical de la zona 4,6. C.2) Radiografía periapical de las zonas correspondientes al 1,5 y 1,6. C.3) Radiografía periapical de la zona 3,5. 


\section{Caso 2}

Paciente varón de 44 años, diagnosticado de VIH en el año 1988. La vía de contagio fue por ADVP. Como antecedentes patológicos el paciente presentó infección diseminada por Mycobacterium Kansasii (Estadio IV-C1), siguiendo durante 24 meses tratamiento tuberculostático. En noviembre del año 1995 presentó una retinitis bilateral con necrosis retiniana por citomegalovirus (herpes viridae) que le ocasionó una ceguera completa. Desde entonces se le efectúa tratamiento por vía intravenosa con Ganciclovir (Cymevene ${ }^{\circledR}$, Roche Farma, Madrid, España) 3 días a la semana. Desde 1991 las cifras de $\mathrm{CD}_{4}$ han estado con valores menores a $100 / \mathrm{mm}^{3}$ y desde 1994 con valores entre $5-20 / \mathrm{mm}^{3}$. El paciente durante los primeros años de enfermedad hizo tratamiento antirretroviral con Zidovudina (Retrovir ${ }^{\circledR}$, GlaxoSmithKline, Madrid, España). A pesar de la severa inmunodepresión que presentaba, una vez controlados los procesos infecciosos agudos relatados, el paciente se mantuvo con un buen estado general, manteniéndose con un peso de $70 \mathrm{~kg}$ y presentando como única limitación la ceguera total.

En abril del año 1997 los valores de $\mathrm{CD}_{4}$ eran del $1 \%$ $\left(8 / \mathrm{mm}^{3}\right)$ y la carga viral de $16.390 \mathrm{c} / \mathrm{ml}$. En ese instante se inició tratamiento con $300 \mathrm{mg}$ de lamivudina (3TC) (Epivir ${ }^{\circledR}$, GlaxoSmithKline, Madrid, España), 40 mg de estavudina (4dt) (Zerit $^{\circledR}$, Bristol-Myers Squibb, Madrid, España) y $600 \mathrm{mg}$ de ritonavir (Norvir ${ }^{\circledR}$, Abbott Laboratorios, Madrid, España). La tolerancia al tratamiento ha sido buena, así como también su eficacia. A partir de ese momento, la evolución clínica del paciente ha sido excelente manteniéndose desde el inicio de esta terapéutica y hasta la actualidad con cargas virales indetectables y cifras de $\mathrm{CD}_{4}$ que se han incrementado de forma espectacular en los últimos años (mayores a $1.000 / \mathrm{mm}^{3}$ y con porcentajes superiores al $25 \%$ ). Actualmente el paciente presenta un aceptable estado de salud, pero su capacidad laboral se ha visto limitada por la ceguera completa que presenta como secuela de la necrosis retiniana. El médico especialista encargado del control de la enfermedad del Hospital Universitario de Bellvitge (L'Hospitalet de Llobregat, Barcelona, España) nos dio su aprobación para llevar a cabo la intervención quirúrgica. La última analítica realizada en febrero de 2005, revelaba una carga viral menor a $100 \mathrm{c} / \mathrm{ml} \mathrm{CD}_{4}$ en un $33 \%\left(1.115 / \mathrm{mm}^{3}\right)$, hemoglobina 16,7 , leucocitos 7.970 y 217.000 de plaquetas. El diagnóstico odontológico fue de edentulismo unitario del 3,6 y 4,6. Las exodoncias habían sido efectuadas hacía 2 años por presentar restos radiculares en ambos molares. El plan de tratamiento que se programó, consistió en la confección de 2 coronas metal-cerámica implantosoportadas mediante 2 implantes ITI ${ }^{\circledR}$ (Straumman, Waldenburg, Suiza) en la mandíbula (2 implantes 4,1x12 mm SLA) (Figura 2). La carga de los implantes se hizo a los 6 meses tras la inserción de los implantes y el tiempo de seguimiento ha sido de 12 meses.

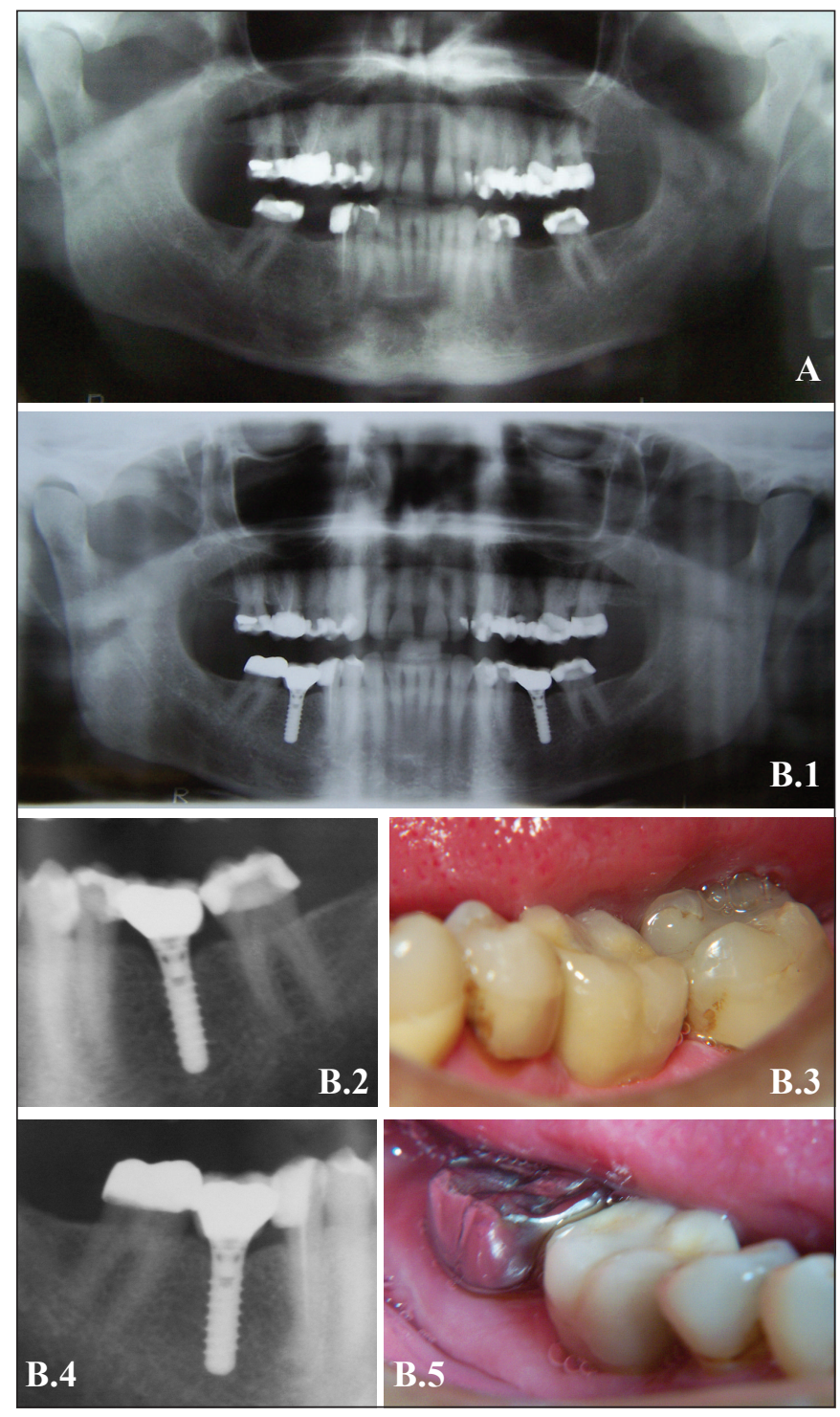

Fig. 2: Caso clínico 2. (A) Ortopantomografía que muestra los espacios edéntulos del 3, 6 y 4,6. (B) Rehabilitación implantosoportada finalizada a los 12 meses tras la inserción de los implantes. B.1) Ortopantomografía con el tratamiento rehabilitador implantosoportado finalizado (2 implantes ITI ${ }^{\circledR}$ en el maxilar inferior de 4,1 $\times 12$ $\mathrm{mm} S L A)$. B.2) Detalle de la ortopantomografía correspondiente a la zona del 3,6. B.3) Imagen clínica de la zona 3, 6 con la prótesis colocada. B.4) Detalle de la ortopantomografía correspondiente a la zona del 4,6. B.5) Imagen clínica de la zona 4,6 con la prótesis colocada. 


\section{Caso 3}

Paciente varón de 43 años, diagnosticado de VIH en el año 1998. La vía de contagio fue sexual. Como antecedentes patológicos el paciente presentó en el mismo año una infección medular por citomegalovirus (CMV) -radiculopolineuropatía - que le provocó una paraplejía espástica que está siendo tratada con infiltraciones cada 6 meses de baclofén (Lioresal ${ }^{\circledR}$, Novartis Farmacéutica, Barcelona, España) y fisioterapia. El paciente había sido intervenido de una rinoseptoplastia en octubre de 2004 por presentar dificultad respiratoria. El paciente no refería tener ninguna alergia medicamentosa conocida. El diagnóstico odontológico fue de edentulismo total del maxilar superior y la presencia de un resto radicular endodonciado del 2.l. La causa de las pérdidas dentales había sido por caries, extracciones efectuadas hacía aproximadamente 2 años. El plan de tratamiento consistió en la extracción qui- rúrgica del resto radicular endodonciado 2.1 y la confección de una prótesis híbrida sobre 6 implantes Brånemark System ${ }^{\circledR}$ MkIII TiUnite ${ }^{\circledR}$ (Nobel Biocare, Göteborg, Suecia) en el maxilar superior (4 implantes de $4,0 \times 13 \mathrm{~mm}$ y 2 implantes de 5,0x13 mm) (Figura 3). Los implantes fueron cargados a los 10 meses tras su inserción, ya que el paciente no acudió a nuestro Servicio durante 4 meses por motivos personales. Es debido a esta eventualidad, el tiempo de seguimiento de este paciente es sólo de 5 meses.

\section{DISCUSIÓN}

Los implantes dentales, son considerados actualmente como una buena opción de tratamiento en los pacientes VIH-positivo pese a que su fiabilidad, no ha sido todavía establecida a largo plazo. A excepción de dos artículos que hemos encontrado en la literatura cientí-
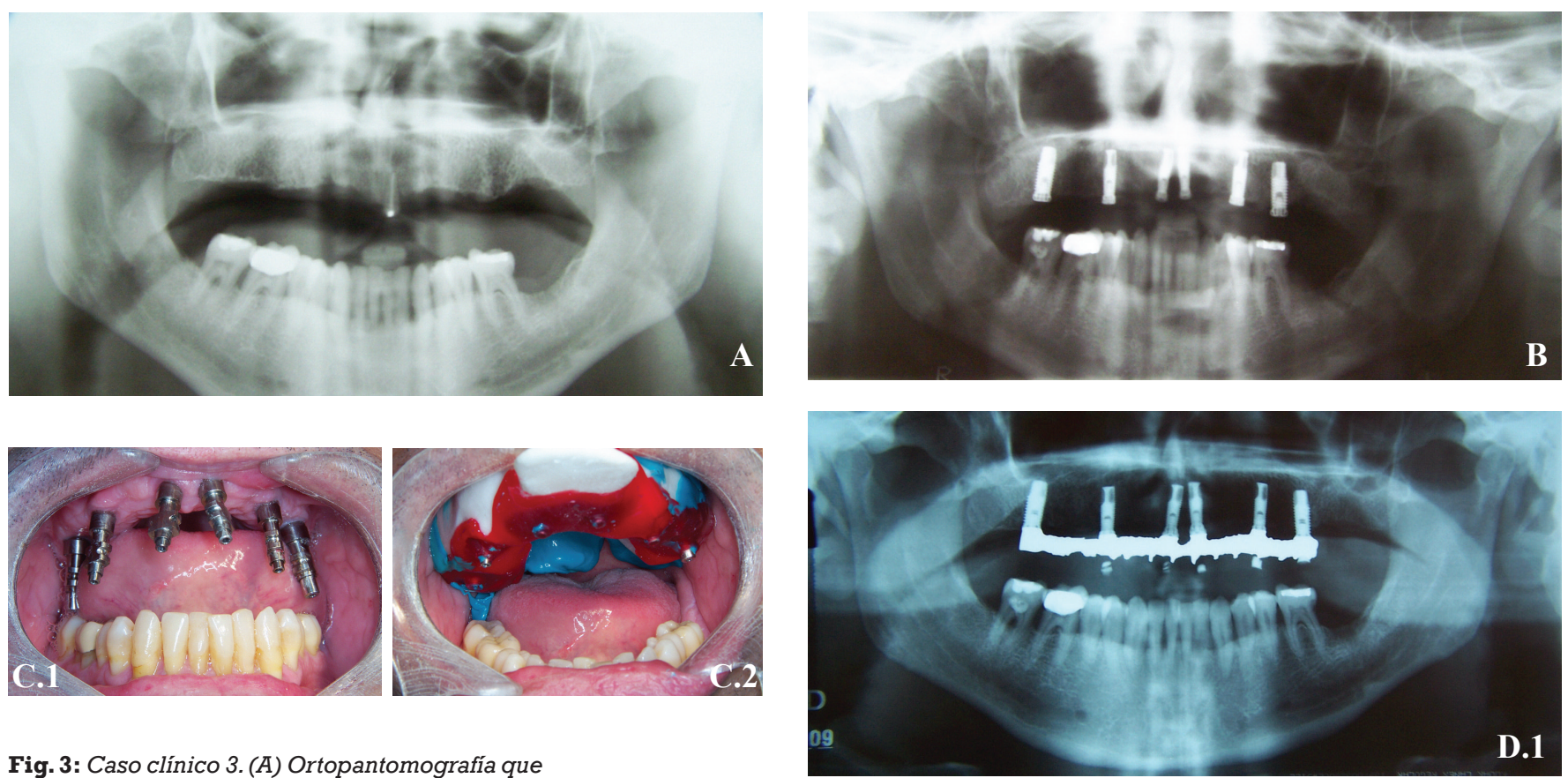

Fig. 3: Caso clínico 3. (A) Ortopantomografía que muestra los espacios edéntulos del maxilar superior e inferior y el resto radicular 2.1 endodonciado. (B) Ortopantomografía realizada a los 10 meses tras la inserción de los 6 implantes Brånemark System ${ }^{\circledR}$ MkIII TiUnite ${ }^{\circledR}$ en el maxilar superior (4 implantes de 4,0x13 mm y 2 implantes $5,0 \times 13 \mathrm{~mm})$. (C) Toma de impresiones durante la fase protésica. C.1.) Aditamentos protésicos. C.2.) Toma de impresión definitiva con cubeta

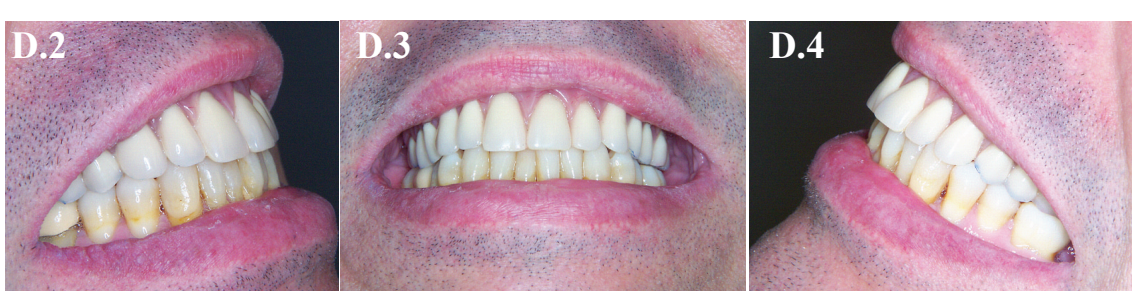
abierta. D. 1) Ortopantomografía con el tratamiento rehabilitador implantosoportado finalizado a los 15 meses de la inserción de los implantes (prótesis híbrida sobre 6 implantes). D.2) Imagen clínica visión lateral derecha. D.3) Imagen clínica visión frontal. D.4) Imagen clínica visión lateral izquierda. 
fica referidos a un implante unitario inmediato (3) y a una rehabilitación bucal completa (4), ningún otro estudio ha sido publicado en relación con la rehabilitación con implantes en pacientes VIH-positivo. En ambos artículos se corrobora la hipótesis que la cirugía bucal menor no aumenta el riesgo de infección en los pacientes VIH-positivo correctamente controlados. En nuestros 3 casos, durante el curso postoperatorio inmediato, no se observó ninguna disminución significativa del marcador $\mathrm{CD}_{4}$ ni de ningún otro parámetro biológico, tal como se describe en la literatura $(3,4)$, obteniéndose una excelente curación de los tejidos blandos y duros.

La literatura publicada que se refiere a las complicaciones de los tratamientos odontológicos y de cirugía maxilofacial en los pacientes VIH-positivo es escasa, tal y como puede observarse en la revisión sistemática realizada por Patton y cols. Estos autores no encuentran ningún estudio, ni caso clínico de cirugía ortognática, tratamientos periodontales o de colocación de implantes dentales en este tipo de pacientes. Únicamente localizan un caso clínico de un paciente VIHpositivo sometido a un tratamiento endodóncico y otros pocos casos sobre exodoncias convencionales. Pese a ello, los autores concluyen que las complicaciones referenciadas sobre los riesgos asociados a los procedimientos invasivos bucales en pacientes VIH son poco relevantes como para contraindicar dichos tratamientos en este tipo de pacientes (5).

Esta falta de información, hace pensar que la incidencia de complicaciones postoperatorias tras tratamientos bucodentales es baja, incluso en los pacientes VIHpositivo tal y como apuntan algunos autores $(5,6)$. No obstante, el número de complicaciones postoperatorias podría aumentar conforme se agrava la infección por VIH (7).

La existencia de una mayor prevalencia de infecciones óseas en los pacientes VIH-positivo que han sufrido traumatismos del área bucofacial también es controvertido. Pese que algunos autores (8) han observado un aumento del número de infecciones óseas tras el tratamiento quirúrgico de fracturas mandibulares en pacientes VIH-positivo asintomáticos (8) existen otros autores que no encuentran diferencias estadísticamente significativas en relación con la incidencia de infecciones postoperatorias en pacientes tratados de fracturas del esqueleto facial (9).

Sin duda alguna, las infecciones postoperatorias son las complicaciones que más temor producen al ciruja- no bucal al tratar este tipo de pacientes. La necesidad de hacer una profilaxis antibiótica especial en los pacientes VIH-positivo sometidos a procedimientos odontológicos invasivos es también motivo de controversia. Respecto a esta cuestión, Patton y cols. son muy firmes al respecto y argumentan que la cobertura antibiótica para prevenir la septicemia desencadenada tras los procedimientos bucales no estaría indicada por el estado seropositivo del paciente (5). En nuestros casos, los tres pacientes recibieron la profilaxis antibiótica habitual previa a cualquier intervención de cirugía implantológica que se administra en nuestro Servicio de Implantología Bucofacial (l gramo por vía oral de amoxicilina (Clamoxyl ${ }^{\circledR}$, GlaxoSmithKline, Madrid, España) 1 hora antes de la intervención quirúrgica o en su defecto, $600 \mathrm{mg}$ por vía oral de clindamicina (Dalacin ${ }^{\circledR}$, Pharmacia Spain, Sant Cugat del Vallés, España) en aquellos pacientes alérgicos a la penicilina y sus derivados). En los otros dos casos clínicos publicados en la literatura, sobre pacientes VIHpositivos sometidos a una rehabilitación implantosoportada $(3,4)$, ambos pacientes tampoco recibieron ningún tipo de profilaxis antibiótica previa, siendo la cobertura antibiótica postoperatoria de $1.500 \mathrm{mg} /$ día de amoxicilina durante 7 días en el caso del implante inmediato postexodoncia (3) y de $900 \mathrm{mg} /$ día de clindamicina durante 7 días en la paciente de la rehabilitación completa del maxilar superior, ya que era alérgica a la penicilina (4). En nuestros tres casos se les administró postoperatoriamente a todos los pacientes $2.250 \mathrm{mg} /$ día de amoxicilina ( 1 comprimido de $750 \mathrm{mg}$ cada 8 horas) puesto que ninguno de ellos era alérgico a la penicilina, siguiendo el protocolo habitual de nuestro Servicio.

En una encuesta efectuada en los EE.UU. sobre el uso de los antibióticos por parte de los odontólogos se observó que los individuos VIH-positivo eran los "pacientes especiales" que mayor cobertura antibiótica recibían antes y después de cualquier procedimiento bucal invasivo, respecto a los pacientes sanos o con otros tipos de patología sistémica (10). En nuestros 3 casos, no variamos el protocolo de profilaxis antibiótica habitual de nuestro Servicio.

La existencia de una reducción postoperatoria del cómputo de los $\mathrm{CD}_{4}$ tras un procedimiento bucal invasivo en un paciente VIH-positivo es un hecho aún no comprobado. La mayoría de autores creen que esta reducción postoperatoria del número de los $\mathrm{CD}_{4}$ se produciría únicamente cuando existen preoperatoriamente procesos inflamatorios en la zona quirúrgica, además de que este hecho podría estar asociado o no al estrés 
al que estarían sometidos estos pacientes $(4,11)$. Por lo tanto, el cómputo de $\mathrm{CD}_{4}$ en un paciente VIH-positivo sometido a un procedimiento bucal invasivo no se vería alterado, siempre y cuando no existiera una infección previa (11). En nuestros casos clínicos, no se observó en ninguno de los pacientes, una disminución del cómputo de los $\mathrm{CD}_{4}$ o de otro parámetro biológico durante el postoperatorio inmediato o a corto - a largo plazo constatado por el médico especialista encargado del control de la enfermedad.

El tiempo de espera para la carga de los implantes en pacientes VIH-positivo tampoco está establecido. Con todo lo comentado anteriormente respecto de la no modificación de las pautas de cobertura antibiótica y tras observar la escasa susceptibilidad a las infecciones postoperatorias en estos pacientes, cabría pensar que la carga de los implantes tampoco debería de diferir del tiempo de carga habitual respecto a los pacientes sanos.

En el caso presentado por Rajnay y cols. los implantes fueron cargados tras 7 meses tras su inserción con un tiempo de seguimiento de 18 meses. En el caso publicado por Baron y cols., el tiempo de seguimiento fue ligeramente mayor 24 meses y la carga de los implantes también se realizó a los 7 meses (3). En este último caso el paciente llevó una prótesis provisional dentosoportada metal-cerámica hasta la confección de la prótesis implantosoportada definitiva (4). En nuestros pacientes, los casos clínicos 1 y 2 los implantes fueron cargados aproximadamente a los 6 meses tras la cirugía de implantes, con un tiempo de seguimiento de 18 y 12 meses respectivamente. El tiempo de seguimiento del tercer paciente es de tan sólo 5 meses, ya que los implantes tuvieron que ser cargados a los 10 meses tras su inserción porque el paciente abandonó el tratamiento durante 4 meses por motivos personales.

Todo lo comentado anteriormente nos lleva a pensar, que durante la colocación de implantes dentales en pacientes VIH-positivo, no parece existir una relación clara entre el fracaso terapéutico (la no osteointegración del implante) y la infección local crónica derivada de la enfermedad, ni que la colocación de implantes endoóseos sea una acción que agrave la enfermedad de base de estos pacientes.

Finalmente hay que destacar que las medidas preventivas utilizadas para la cirugía implantológica no deberían de diferir de las empleadas para la cirugía bucal de un paciente sano, excepto en la recomendación de la utilización de un doble guante, que reduciría del 60 al $80 \%$ las posibilidades de contagio de la infección por VIH en caso de pinchazo accidental (12).

\section{CONCLUSIONES}

Los implantes dentales pueden ser considerados como una buena opción de tratamiento, alternativa a las prótesis removibles, en los pacientes VIH-positivo bien controlados, aunque son necesarios más estudios prospectivos con muestras más amplias y con un mayor tiempo de seguimiento, antes de que este tipo de tratamiento pueda ser aceptado de forma rutinaria.

\section{AGRADECIMIENTOS}

Este estudio ha sido realizado por el grupo de investigación "Patología y Terapéutica Odontológica y Maxilofacial" del Instituto UB-IDIBELL con el soporte económico del convenio docente-asistencial de Cirugía Bucal entre la Universitat de Barcelona, el Consorci Sanitari Integral y el Servei Català de la Salut de la Generalitat de Catalunya.

\section{BIBLIOGRAFÍA}

1. Vilaseca J, Arnau JM, Bacardi R, Mieras C, Serrano A, Navarro C. Kaposi's sarcoma and toxoplasma gondii brain abscess in Spanish homosexual. Lancet 1982; 1:572.

2. Brugal MT, Caylà JA, Díez E, Fernández S, García de Olalla P, Plasencia A, Carlota R, Villalbí JR. Epidemiología. En:VIH/SIDA. Barcelona 1981-2002. Barcelona: Instituto Municipal de Salud Pública. Ajuntament de Barcelona, 2002:9-20.

3. Rajnay Z. Immediate placement of an endosseous rootform implant in an HIV-positive patient: Report of a case. J Periodontol 1998;69:1167-71.

4. Baron M, Gritsch F, Hansy A.M, Haas R. Implants in an HIV-positive patient: A case report. Int J Oral Maxillofac Implants 2004;19:425-30.

5. Patton LL, Shugars DA, Bonito AJ. A systematic review of complication risks for HIV-positive patients undergoing invasive dental procedures. J Am Dent Assoc 2002; 133:195-203. 
6. Glick M, Abel SN, Muzyka BC, Delorenso M. Dental complications after treating patients with AIDS. J Am Dent Assoc 1994;125:296-301.

7. Dodson TB, Perrot DH, Gongloff RK, Kaban LB. Human immunodeficiency virus serostatus and the risk of postextraction complications. Int J Oral Maxillofac Surg 1994;23:100-3.

8. Schmidt B, Kearns G, Perrott D, Kaban LB. Infection following treatment of mandibular fractures in human immunodeficiency virus seropositive patients. J Oral Maxillofac Surg 1995; 1 1:34-9.

9. Martínez-Gimeno C, Acero-Sanz J, Martín-Sastre R, Navarro-Vila C. Maxillofacial trauma: Influence of HIV infection. J Craniomaxillofac Surg 1992;20:297-302.

10. Epstein JB, Chong S, Le ND. A survey of antibiotic use in dentistry. J Am Dent Assoc 2000;131:1600-9.
11. Harrison W, Lewis C, Lavy C. Wound healing after implant surgery in HIV-positive patients. J Bone Joint Surg Br 2002;84:802-6.

12. Gerberding JL, Schecter WP. Surgery and AIDS. Reducing the risk. J Am Med Assoc 1991;265:1572-3.

\section{CORRESPONDENCIA}

Dr. Cosme Gay-Escoda

Centro Médico Teknon

Instituto de investigación UB-IDIBELL.

Vilana, 12

08022 Barcelona

Teléfono: 9340242 74; Fax: 933933070

E-mail: cgay@ub.edu

Website: www.gayescoda.com 